\title{
AN APPLICATION OF A THEOREM OF R. E. ZINK
}

\author{
H. E. WHITE, JR. ${ }^{1}$
}

\begin{abstract}
In $\S 1$ we discuss a measure theoretic analogue of Blumberg's theorem; in $\$ 2$ we discuss a topological analogue of the Saks-Sierpinski theorem.
\end{abstract}

1. In this section we discuss a measure theoretic analogue of Blumberg's theorem [7, 1.2]. Suppose $(X, \delta, \mu)$ is a totally finite measure space $[2$, p. 73], and $\mu^{*}$ is the outer measure engendered by $\mu$. We consider the following statement.

1.1. For every real valued function $f$ defined on $X$, there is a subset $D$ of $X$ such that $\mu^{*}(D)=\mu(X)$ and $f \mid D$ is measurable $(\mathcal{S} \cap D)$, where $\delta \cap D=$ $\{S \cap D: S \in \mathcal{S}\}$.

Actually 1.1 is equivalent to a special case of Blumberg's theorem. Let $(X$, $\left.\delta_{c}, \mu_{c}\right)$ denote the completion of $(X, \delta, \mu)$. By [3, p. 88], there is a topology $\mathcal{T}\left(\mu_{c}\right)$ on $X$ such that (a) if $U \in \mathcal{T}\left(\mu_{c}\right)$ and $U \neq \varnothing$, then $U \in \mathcal{S}_{c}$ and $\mu_{c}(U)>0$; and (b) if $A \in \mathcal{S}_{c}$, then there is $U$ in $\mathcal{T}\left(\mu_{c}\right)$ such that $U \subset A$ and $\mu_{c}(A \sim U)=0$. It is easily verified that 1.1 holds for $(X, \delta, \mu)$ if and only if Blumberg's theorem holds for $\left(X, \mathcal{T}\left(\mu_{c}\right)\right)$.

In $[7,2.1]$ it is shown that

1.2. every subset of the closed unit interval $I$ of cardinality $<2^{\aleph_{0}}$ has Lebesgue measure zero,

then 1.1 is false for $(I, \mathscr{B}, m)$, where $m$ denotes Lebesgue measure on the collection $\Re$ of Borel subsets of $I$. In this section we show that the following statement is a consequence of [9, Theorem 9].

1.3. TheOREM. Suppose 1.1 is false for $(I, \Re, m)$. Then 1.1 is false for every separable, nonatomic measure space $(X, \delta, \mu)$.

REMARK. It follows that, in this case, there is $f: X \rightarrow R$ such that, if $D$ is a subset of $X$ for which $f \mid D$ is measurable $\left(\delta \cap D\right.$ ), then $\mu^{*}(D)=0$.

If $2^{\kappa_{0}}=\aleph_{1}$, then clearly 1.2 holds; therefore, in this case, 1.1 is false for every separable, nonatomic measure space. However, there is a weaker statement-called Martin's axiom-which implies that 1.2 is true $[5, \S 4]$.

Presented to the Society May 17, 1976; received by the editors May 29, 1976.

AMS (MOS) subject classifications (1970). Primary 28A20.

Key words and phrases. Totally finite nonatomic separable measure space, Saks-Sierpinski theorem.

'During the period in which this research was done, the author held a Visiting Postdoctoral Fellowship from the Institute for Medicine and Mathematics.

(1) American Mathematical Society 1977 
MARTIN's AXIOM. If $C$ is any collection of fewer than $2^{\mathrm{N}_{0}}$ dense open subsets of a compact Hausdorff space which satisfies the countable chain condition, then $\cap \mathcal{C} \neq \varnothing$.

1.4. Corollary. Suppose Martin's axiom holds. If $(X, \mathcal{T})$ is a quasi-regular [4, p. 157] Baire space which admits a separable, nonatomic category measure [4, p. 156] $\mu$, then Blumberg's theorem does not hold for $(X, \mathcal{T})$.

This corollary follows from 1.3 because Blumberg's theorem holds for $(X$, $\mathcal{T})$ if and only if 1.1 holds for $(X, \delta, \mu)$, where $\delta$ denotes the collection of all subsets of $(X, \mathcal{T})$ having the property of Baire.

1.5. EXAMPLES. (1) Suppose $\mathscr{D}$ denotes the density topology on the real line $R[7,2.1]$. Then the Stone-Čech compactification of $(R, \mathscr{D})$ satisfies the hypothesis of 1.4 because $(R, \mathcal{Q})$ does [3, p. 91].

(2) Suppose $(S, \mathcal{Q})$ denotes the Stone space of $\mathcal{L} / \mathcal{K}$, where $\mathcal{L}$ is the collection of all Lebesgue measurable subsets of $I$ and $\mathscr{K}=\{A \in \mathcal{L}: m(A)$ $=0\}$. Then $(S, \mathscr{Q})$ satisfies the hypothesis of $1.4[3, \mathrm{p} .91]$.

REMARK. It is proven in [6] that if $2^{\kappa_{0}}=\kappa_{1}$, then Blumberg's theorem does not hold for $(S, \mathcal{Q})$. The preceding provides a measure theoretic proof of this statement.

Proof of 1.3. We shall show that 1.3 follows easily from the next statement, which is a result from [9].

1.6. Theorem (R. E. ZINK). Suppose $(X, \mathcal{S}, \mu)$ is a separable, nonatomic, totally finite measure space such that $\mu(X)=1$. Then there is a function $T$ : $X \rightarrow I$ such that (a) $T^{-1}[\mathscr{B}] \subset \mathcal{S}$; (b) if $B \in \mathscr{B}$, then $\mu\left(T^{-1}[B]\right)=m(B)$; and (c) if $S \in \mathcal{S}$, then there is $B$ in $\mathscr{B}$ such that $\mu\left(S \triangle T^{-1}[B]\right)=0$, where " $\triangle$ " denotes symmetric difference.

To prove 1.3, we assume that 1.1 holds for some separable, nonatomic, totally finite measure space $(X, \mathcal{S}, \mu)$. We assume $\mu(X)=1$, and that $T$ is as described in 1.6. Suppose $f_{0}: I \rightarrow R$, and let $f=f_{0} \circ T$. Then there is a subset $D_{1}$ of $X$ such that $\mu^{*}\left(D_{1}\right)=1$ and $f \mid D_{1}$ is measurable $\left(\delta \cap D_{1}\right.$ ). Because 1.6 (c) holds, there is a subset of $D$ of $D_{1}$ such that $\mu^{*}(D)=1$ and $f \mid D$ is measurable $T^{-1}[\mathscr{B}] \cap D$. Then $f \mid T^{-1}[T[D]]$ is measurable $\left(T^{-1}[\mathscr{B}] \cap\right.$ $\left.T^{-1}[T[D]]\right)$, and $f_{0} \mid T[D]$ is measurable $\mathscr{B} \cap T[D]$. Because $1.6(\mathrm{~b})$ holds, $m^{*}(T[D]) \geqslant \mu^{*}(D)=1$; hence 1.1 holds for $(I, \mathscr{B}, m)$.

Questions. (1) Is it consistent with $\mathrm{ZF}+\mathrm{AC}$ that 1.1 holds for $(I, \Re, m)$ ?

(2) Does 1.3 remain true if the word "separable" is deleted from the hypothesis?

2. In [9], it is shown that the following statement, known as the Saks-Sierpinski theorem, holds for every totally finite measure space $(X, \mathcal{S}, \mu)$.

2.1. For évery real valued function $f$ defined on $X$, there is a function $g$ : $X \rightarrow R$ which is measurable $(\delta)$ such that, for every positive number $\varepsilon$,

$$
\mu^{*}(\{x:|f(x)-g(x)|<\varepsilon\})=\mu(X) .
$$


A topological analogue of 2.1 provides another characterization of Baire spaces.

2.2. THEOREM. The following statements are equivalent for any topological space $(X, \mathcal{T})$.

(a) $(X, \mathcal{T})$ is a Baire space.

(b) For every real valued function $f$ defined on $X$, there is a function $g$ such that: (i) domain $g$ is a dense $G_{\delta}$ subset of $X$; (ii) $g$ is continuous; and (iii) for every positive number $\varepsilon$, the set $\{x \in$ domain $g:|f(x)-g(x)|<\varepsilon\}$ is dense in $X$.

(c) For every real valued function $f$ defined on $X$, there is a function $h$ defined on $X$ such that: (i) $h$ is Borel measurable; and (ii) for every positive number $\varepsilon$, there is a dense subset $D_{\varepsilon}$ of $X$ such that $h \mid D_{\varepsilon}$ is continuous and $D_{\varepsilon} \subset\{x$ : $|f(x)-h(x)|<\varepsilon\}$.

Proof. Obviously (b) implies (c). If $[7,1.5]$ is modified by replacing $Y$ by $R$ in (3), then the resulting statement is true, and its proof is very similar to the proof of $[7,1.5]$. It follows from this modified statement that (c) implies (a). Finally, the proof of the Saks-Sierpinski theorem, which is given in [9, §4], when translated into topological terms, establishes that (a) implies (b), provided the following statement is substituted for Lemma B of [9].

Lemma B'. Suppose $f$ is a real valued function defined on the Baire space $X$, and $Y$ is a dense subspace of $X$ such that $Y$ is a Baire space. For every positive number $\varepsilon$, there is a function $f_{\varepsilon}$ such that: (i) domain $f_{\varepsilon}$ is a dense open subset of $X$; (ii) $f_{\varepsilon}$ is continuous; and (iii) if $X_{\varepsilon}=\left\{x \in\right.$ domain $f_{\varepsilon}$ : $\left.\left|f(x)-f_{\varepsilon}(x)\right|<\varepsilon\right\}$, then $X_{\varepsilon} \cap Y$ is dense in $X$ and $X_{\varepsilon} \cap Y$ is a Baire space.

The proof of Lemma $B^{\prime}$ is quite similar to the proof that (1) implies (3) of $[7,1.5]$, and is omitted.

REMARKS. (1) It is clear from the proof of 2.2 that:

(a) The function $g$ (resp. $h$ ) can be chosen so that for every positive number $\varepsilon$, the set $\{x \in$ domain $g:|f(x)-g(x)|<\varepsilon\}$ (resp. $D_{\varepsilon}$ ) is a dense Baire subspace of $X$.

(b) If $f$ is bounded, then function $g$ (resp. $h$ ) can be chosen to be bounded.

(c) If $X$ is completely regular and satisfies the countable chain condition, then $h$ can be chosen to be a Baire function.

(2) The Saks-Sierpinski theorem, when $\mu$ is complete, is a special case of 2.2 (apply 2.2(c) to the Baire space $(X, \tilde{\mathcal{T}}(\mu))$ ).

(3) Suppose $(X, \widetilde{T})$ is an extremally disconnected [1, p. 22] Baire space. It follows from 2.2(b) and [1, p. 96] that for every bounded, real valued function $f$ defined on $X$, there is a continuous function $g: X \rightarrow R$ such that, for every positive number $\varepsilon$, the set $\{x:|f(x)-g(x)|<\varepsilon\}$ is dense in $X$. In particular, the preceding statement holds for the space $\left(S, \vartheta^{2}\right)$ of 1.5(2). However, if $2^{\kappa_{0}}=\kappa_{1}$, then there is a bounded real valued function $f_{0}$ defined on $S$ such 
that $\left\{x: f_{0}(x)=g(x)\right\}$ is nowhere dense in $S$ for every continuous real valued function $g$ defined on $S$.

\section{REFERENCES}

1. L. Gillman and M. Jerison, Rings of continuous functions, Van Nostrand, Princeton, N. J., 1960. MR 22 \#6994.

2. P. R. Halmos, Measure theory, Van Nostrand, Princeton, N. J., 1950. MR 11, 504.

3. J. C. Oxtoby, Measure and category, Springer-Verlag, New York, 1971.

4. Spaces that admit a category measure, J. Reine Angew. Math. 205 (1960/61), 156-170. MR 25 \# 4054; 26, p. 1543.

5. F. Tall, The density topology (preprint).

6. W. A. R. Weiss, A solution to the Blumberg problem, Bull. Amer. Math. Soc. 81 (1975), 957-958.

7. H. E. White, Jr., Topological spaces in which Blumberg's theorem holds, Proc. Amer. Math. Soc. 44 (1974), 454-462. MR 49 \#6130.

8. Some Baire spaces for which Blumberg's theorem does not hold, Proc. Amer. Math. Soc. 51 (1975), 477-482.

9. R. E. Zink, On the structure of measure spaces, Acta Math. 107 (1962), 53-71. MR 25 \#4060.

671 S. Eureka Avenue, Columbus, Ohio 43204 